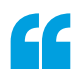

This is a key step between the first demonstrations of atomicresolution AFM imaging of flat molecules and the possibility of unravelling the structure of complex 3D molecules
ATOMIC FORCE MICROSCOPY

\section{Seeing in 3D}

Atomic force microscopy (AFM) and machine learning, combined, can unveil the structure of 3D molecules of any size and configuration, as Peter Liljeroth, Adam Foster and colleagues report in Science Advances.

AFM is a powerful technique that has elucidated a wide range of physical, biological and chemical processes; with the addition of a $\mathrm{CO}$ molecule on the tip, it also offers very high-resolution imaging. However, the interpretation of the data is often not straightforward, because the measured images are a convolution of the surface topography and induced changes in the tip structure. For this reason, so far most AFM studies have focused on planar molecules, for which the interpretation of the images is simple, or can be clarified using atomistic models.

Using AFM to image unknown 3D molecules would be very relevant for fields ranging from biochemistry to drug discovery, but for 3D systems

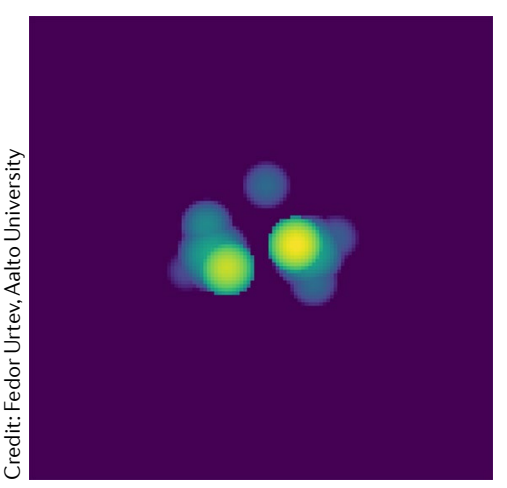

image interpretation is most often prohibitively complex. Particularly, when there are open questions about the nature of the system, standard atomistic methods become insufficient. Machine learning can be the answer.

"We have created a machine learning infrastructure that can identify the signatures of $3 \mathrm{D}$ molecules based on a stack of AFM images acquired at different tipmolecule distances", summarizes Foster. Because experiments are time consuming and the resulting images are not labelled a priori, the machine learning algorithm was trained on simulated AFM data from 134,000 small organic molecules.

As the CO molecule at the end of the tip is flexible, the image contrast is not trivially related to the atomic positions. The authors used machine learning to invert the function describing the imaging process and thus obtain the molecular structure.

"We wanted to do something similar to single-particle reconstruction, a transmission electron microscopy (TEM) technique in which a number of TEM projections of a given structure are combined to produce a 3D image", explains Liljeroth. "The original idea was to image several different adsorption configurations of a molecule and then somehow combine these images into its 3D structure. I had initially no idea what the 'somehow' would be, but that is where Adam, machine learning and simulated AFM images came in, and the original idea evolved into identifying individual molecules".

To validate the method, the authors applied it to the interpretation of experimental COfunctionalized AFM images of camphor molecules on a copper surface. Camphor can adopt multiple adsorption geometries on copper, thus this is a good test system. The machine learning algorithm correctly identified all the different configurations, a feat that would have been close to impossible for human experts. "This is a key step between the first demonstrations of atomic-resolution AFM imaging of flat molecules and the possibility of unravelling the structure of complex 3D molecules," comments Foster.

The approach is immediately applicable to many complex molecular systems, but it can also be improved to make it more powerful and far reaching. "Apart from the information on the atomic positions, we have some ideas on how to get information on the atom types, and we are planning to experiment with more complicated biomolecules to try to push their structural characterization," concludes Liljeroth. "Finally, there would be a lot to do for automatizing the experiments, starting from the tip preparation".

Giulia Pacchioni

ORIGINAL ARTICLE Alldritt, B. et al. Automated structure discovery in atomic force microscopy. Sci. Adv. 6, eaay6913 (2020) 